\section{Response to "Association between statin use and ischemic stroke or major hemorrhage in patients taking dabigatran for atrial fibrillation"}

We thank Dr. Haertter and colleagues for their letter. ${ }^{1}$ We conducted a case-control study nested within a cohort of patients initiating dabigatran therapy. ${ }^{2}$ Following adjustment for baseline variables, we found a higher risk of bleeding among patients treated with simvastatin and lovastatin relative to other statins. Because randomized trials are typically not designed to examine outcomes of specific drug interactions and patients in such studies usually differ in important ways from those who receive interacting drugs in clinical practice, post-marketing observational studies such as ours provide complementary information that supports safe prescribing.

Our findings are consistent with prior research showing that $\mathrm{P}$-glycoprotein inhibitors, including verapamil, increase systemic exposure to dabigatran. ${ }^{3-5}$ Although the magnitude of change following concomitant dabigatran-verapamil administration was lower in the RE-LY study, Dr. Haertter's own work shows that changes in dabigatran concentrations following verapamil exposure vary with the timing and formulation of verapamil used. ${ }^{3}$ The timing of administration of dabigatran relative to verapamil in RE-LY is unknown and may account for this discrepancy, particularly because the protocol was amended in February 2008 to caution about combined use. ${ }^{6}$
Further, a greater increase in dabigatran exposure was observed among participants receiving verapamil in the RECOVER study. ${ }^{7}$

Although it is true that statins generally cause little to no change in digoxin exposure, dabigatran may be susceptible to larger relative changes in systemic exposure following inhibition of intestinal P-glycoprotein because of its lower bioavailability (3\%-7\% v. $75 \%)^{4,8}$ The finding that statins were not associated with an increased risk in RE-LY likely reflects inter-study differences in design and population. Specifically, we compared outcomes between statin types - the question of interest rather than in treated versus untreated patients. In addition, the RE-LY study was neither designed nor powered to evaluate this interaction.

Finally, patients in our study may have been at higher risk for hemorrhage than RE-LY participants because of older age (82 yr v. $71 \mathrm{yr}$ ) and lack of exclusions related to the risk of bleeding. Our study reflects outcomes of a drug-drug interaction under "real-world" conditions of dabigatran use. Our primary message to clinicians and pharmacists is that when patients treated with dabigatran require statin therapy, it is prudent to avoid a drug that may increase the risk of bleeding, particularly in high-risk patients such as those in our study.

\section{Tony Antoniou PhD}

Department of Family and Community Medicine; Li Ka Shing Knowledge Institute, St. Michael's Hospital; University of Toronto; Institute for Clinical Evaluative Sciences, Toronto, Ont.

\section{Muhammad Mamdani PharmD MPH}

Li Ka Shing Knowledge Institute,

St. Michael's Hospital; University of Toronto; Institute for Clinical Evaluative Sciences; Applied Health Research Centre, St. Michael's Hospital, Toronto, Ont.; King Saud University, Riyadh, Saudi Arabia

\section{David Juurlink MD PhD}

University of Toronto; Institute for Clinical Evaluative Sciences, Toronto, Ont.

- Cite as: CMAJ 2017 February 27;189:E326. doi: $10.1503 / \mathrm{cmaj} .732638$

\section{References}

1. Haertter S, Huang F, Franca LR. Association between statin use and ischemic stroke or major hemorrhage in patients taking dabigatran for atrial fibrillation [letter]. CMAJ 2017;189:E325.

2. Antoniou T, Macdonald EM, Yao Z, et al. Association between statin use and ischemic stroke or major hemorrhage in patients taking dabigatran for atrial fibrillation. CMAJ 2017;189:E4-10.

3. Härtter S, Sennewald R, Nehmiz G, et al. Oral bioavailability of dabigatran etexilate (Pradaxa $\left({ }^{\circledR}\right)$ ) after co-medication with verapamil in healthy subjects. Br J Clin Pharmacol 2013;75:1053-62.

4. Horn JR, Hansten PD. Dabigatran: a new oral anticoagulant. Pharm Times 2010;76:59.

5. Gordon LA, Kumar P, Brooks KM, et al. Antiretroviral boosting agent cobicistat increases the pharmacokinetic exposure and anticoagulant effect of dabigatran in HIV-negative healthy volunteers. Circulation 2016;134:1909-11.

6. Boehringer Ingelheim. Addendum to clinical review for NDA 22-512. Silver Spring (MD): US Food and Drug Administration. 2010. Available: www. accessdata.fda.gov/drugsatfda_docs/nda/2010 /022512orig1s000medr.pdf (accessed 2016 Dec. 18).

7. Assessment report - Pradaxa. London (UK): European Medicines Agency; 2014. Available: www. ema.europa.eu/docs/en_GB/document_library/ EPAR_-_Assessment_Report_-_Variation/human /000829/WC500169948.pdf (accessed 2016 Dec. 18).

8. Binnion PF. A comparison of the bioavailability of digoxin in capsule, tablet, and solution taken orally with intravenous digoxin. J Clin Pharmacol 1976;16:461-7.

Competing interests: None declared. 